\title{
Composition and lattice structure of fivefold twinned nanorods of silver
}

\author{
H. Hofmeister ${ }^{\mathrm{a}, *}$, S.A. Nepijko ${ }^{\mathrm{b}, 1}$, D.N. Ievlev ${ }^{\mathrm{b}}$, W. Schulze ${ }^{\mathrm{b}}$, G. Ertl $^{\mathrm{b}}$ \\ a Max-Planck-Institut für Mikrostrukturphysik, Weinberg 2, D-06120 Halle, Germany \\ ${ }^{\mathrm{b}}$ Fritz-Haber-Institut der Max-Planck-Gesellschaft, Faradayweg 4-6, D-14195 Berlin, Germany
}

Received 17 September 2001; accepted 2 October 2001

Communicated by R. Kern

\begin{abstract}
Multiply twinned silver particles of rod-like shape and nanometer dimensions prepared by inert-gas aggregation technique have been studied by high-resolution electron microscopy. These pentagonal nanorods exhibit aspect ratio between 1.8 and 6 with the length of their fivefold axis ranging from 22 to $132 \mathrm{~nm}$. Digital image processing and evaluation was utilised to characterise composition and lattice structure of the nanorods. Measuring the spacings of lattice plane fringes of nanorod subunits in various orientations revealed no deviation from the face centred cubic lattice type. There was also no indication of extended lattice defects found. Instead, a certain extent of non-regular lattice distortions recognised in the surface regions of the nanorods apparently is an effective means to achieve sufficient space filling. (C) 2002 Elsevier Science B.V. All rights reserved.
\end{abstract}

PACS: 61.16.d; 61.46. +w; 61.72.Mm; 81.15.Ef

Keywords: A1. Characterization; A1. Electron microscopy; A3. Physical vapor deposition processes; B1. Metal; B1. Nanomaterials

\section{Introduction}

Fivefold twinning of thin films and small particles of nanometer dimensions is widespread in transition metals with face-centred cubic (fcc) crystal lattice. One of the main characteristics of multiply twinned particles (MTPs) is their nearly spherical shape achieved by means of their

\footnotetext{
*Corresponding author. Tel.: +49-345-5582677; fax: +49345-5511223.

E-mail address: hof@mpi-halle.mpg.de (H. Hofmeister).

${ }^{1}$ Permanent address: Institute of Physics, National Academy of Sciences of Ukraine, pr. Nauki 46, $03039 \mathrm{Kiev}$, CIS/Ukraine.
}

composition of tetrahedral subunits. These subunits must slightly deviate from the shape of tetrahedra to avoid spatial discontinuities. Different from single crystalline particles, the common growth form of which is a cuboctahedron, MTPs are bounded by triangular $\left\{\begin{array}{lll}1 & 1 & 1\end{array}\right\}$ faces of tetrahedra only. The minimisation of surface energy by approaching a sphere is most effectively realised with the icosahedron, consisting of 20 twin-related tetrahedra arranged around one common corner. For the decahedron (pentagonal bipyramid), consisting of 5 twin-related tetrahedra arranged around one common edge, that is the fivefold axis of the particle, a specific set of truncations was 
observed resulting in a more spherical shape [1]. The corresponding strong faceting model is derived from a modified Wulff construction [2]. That means, decahedral particles must not necessarily be bounded by $\left\{\begin{array}{lll}1 & 1\end{array}\right\}$ only, but may be stabilised by the presence of $\{100\}$ faces. For both types of MTPs, decahedra and icosahedra, size limits are predicted based on thermodynamic considerations [3]. Experimentally observed fivefold twinned structures, however, not only exceed frequently these size limits [4], but also exhibit distinct deviations from the nearly spherical shape into various types of rod-like [5] or even star-like particle shape [6]. Generally, such particle morphologies occur when there is very strong kinetic control of the growth [7].

Multiply twinned rod-like or needle-shaped particles, sometimes also named whiskers or dendrites, of more or less established growth anisotropy, reflected by the corresponding aspect ratio of the elongated particles, have been known since decades. The materials and processes involved may be summed up in brief. Melmed and Hayward [8] observed Ni, Fe and Pt whiskers of pentagonal shape grown from the vapour phase on tungsten substrates. Although this was only the second paper on multiply twinned structures, it gave a model consisting of five fcc twin-related subunits with only slight lattice distortions. Ogburn et al. [9] reported the observation of pentagonal dendrites of $\mathrm{Cu}$ grown from the vapour phase. DeBlois [10,11] found pentagonal $\mathrm{Ni}$ whiskers by hydrogen reduction of $\mathrm{NiBr}_{2}$. Digard et al. [12] clearly showed the presence of particles of pentagonal prism shape capped by pentagonal pyramids, besides various multiply twinned structures formed by electrodeposition of Ag. Hayashi et al. [13] reported on particles of rod-like shape obtained by gas evaporation technique of $\mathrm{Pd}$ which exhibit characteristic features of fivefold twinning. Brieu and Gillet [14-16] studied by means of TEM tilt series the internal structure of rod-like decahedra grown by reduction of $\mathrm{NiBr}_{2}$ in organic solution. Elongated copper particles of several hundred nanometer length having fivefold axes and aspect ratios up to around 10 were observed by Smith and Gai [17] using selected area electron diffraction to identify the multiply twinned structure. Dahmen and Westmacott [18] found pentagonal, needle-shaped precipitates of $\mathrm{Ge}$ in aluminium matrix. Millers and Kuzjukévics [19] observed fivefold twinned rod-like particles of CVD-grown TiN having additional re-entrant $\left\{\begin{array}{lll}1 & 1 & 1\end{array}\right\}$ faces at their prism edges. Arita et al. [20] found $\mathrm{Yb}$ fivefold twinned pentagonal rods from inert-gas aggregation synthesis. Romanov et al. [21] observed multiply twinned whiskers of CdTe. Lisiecki et al. [22] studied very recently the internal structure of rodlike particles of $\mathrm{Cu}$ of truncated decahedron shape formed by hydrazine reduction of an organometallic copper compound. A short report on pentagonal nanorods of silver prepared by inertgas aggregation was given by Nepijko et al. [23].

Metal nanorods have a great potential to enhance many linear and non-linear processes such as surface Raman scattering, photoluminescence [24] and second harmonic generation. With gold nanorods fluorescence enhancement of several orders of magnitude, up to one million, has been reported [25]. To stress in more detail the issue of the composition of rod-like particles by stacking of twin-related subunits we applied highresolution electron microscopy and digital image processing. Based on corresponding results we treat somewhat quantitatively the crystal lattice of these subunits arranged around a common fivefold axis and the potential of space filling of distorted fcc subunits, which has been a matter of debate since decades [4].

\section{Experimental procedure}

Ag particles were produced by the inert-gas aggregation technique in an ultrahigh vacuum equipment and deposited on $\mathrm{NaCl}\left(\begin{array}{lll}0 & 0 & 1\end{array}\right)$ cleavage planes [26]. Silver was evaporated from a Knudsen cell in an argon atmosphere and the mixture of gas and metal atoms was transferred to a nucleation zone. In this region of laminar flow particles were formed at high enough supersaturation and grew according to the applied conditions as reported in more detail by Frank et al. [26]. After removal of the argon gas, particles of the cluster beam were collected on a sampling probe where the substrate 
crystal was mounted. Subsequently, this substrate crystal was coated by a thin amorphous carbon film of about $10 \mathrm{~nm}$ thickness and removed out of the chamber.

The carbon films containing the as-deposited silver particles were detached from the substrate by floating in distilled water and mounted on copper microgrids. Electron microscopy characterisation, including HREM and SAED, was done by means of a Philips CM 200 FEG operating at $200 \mathrm{kV}$. A rotation/tilt sample holder (GATAN) was employed to adjust a well-defined particle orientation with respect to the electron beam. The spacings of lattice plane fringes, their angular and spatial relation were determined from digitised electron micrographs by digital image processing and evaluation using the Digital Micrograph
(GATAN) and NIH Image [27] software. Mostly, the Fourier transform procedure was employed to obtain a reciprocal-space representation, that is a diffractogram, of an image.

\section{Results}

Besides a background of densely arranged particles of nearly spherical shape and very small size (around 2-4 nm) inert-gas aggregation synthesis produced another class of deposited $\mathrm{Ag}$ particles having spheroidal and rod-like shape on which we will focus in the present work. In an overview at low magnification Fig. 1(a) shows a typical collection of the second class of particles within which a rod-like one is marked by an arrow.
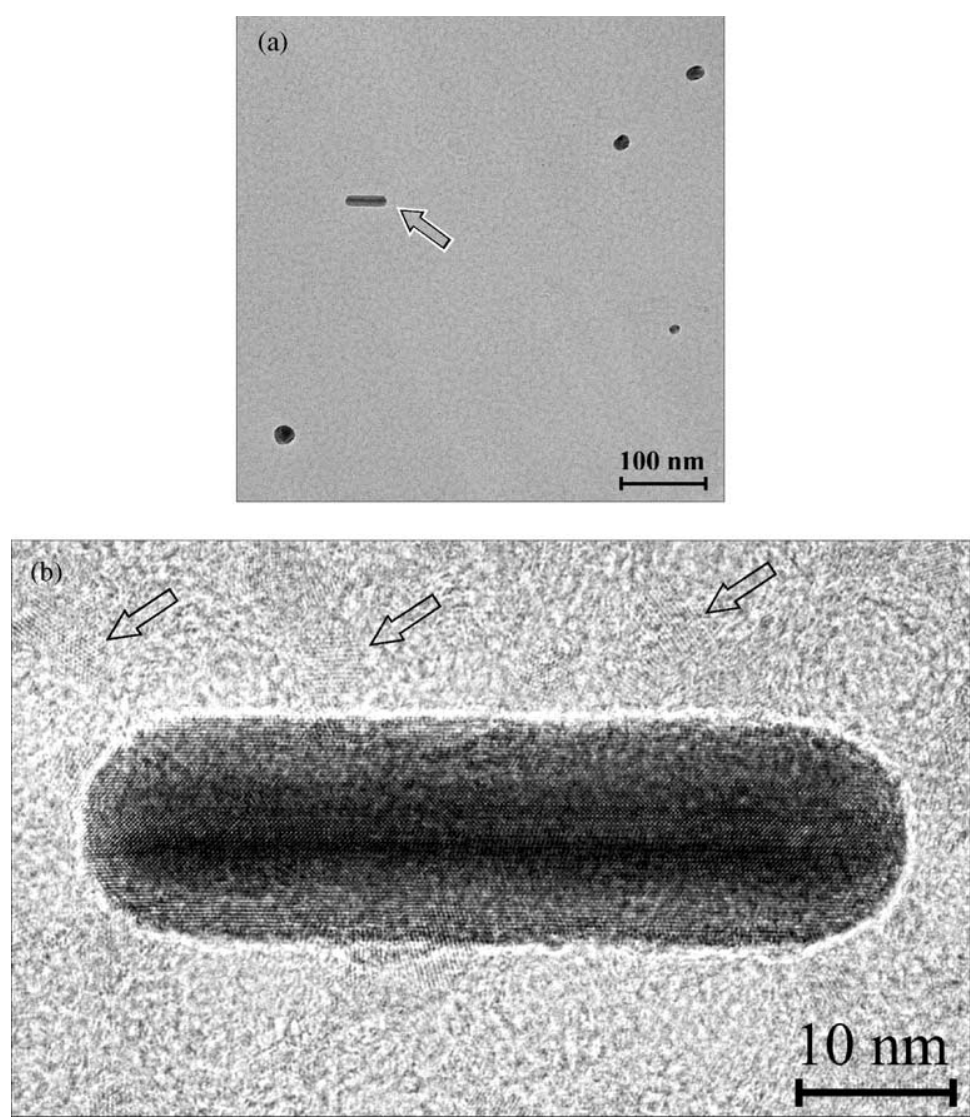

Fig. 1. Overview on a typical collection of particles of spheroidal and rod-like shape (a) and (b) HREM image of the rod-like particle marked by an arrow in $1(\mathrm{a})$. 
These rod-like particles exhibit a certain variation of size and elongation. The longitudinal extension varies from nearly 22 to about $132 \mathrm{~nm}$, whereas the lateral extension ranges from about $8-32 \mathrm{~nm}$. Accordingly, the aspect ratio varies from about 1.8 to nearly 6 . The typical rod-like shape may be recognised from Fig. 1(b) featuring the particle marked in Fig. 1(a). A careful inspection of this HREM image reveals a rather complicated contrast of superimposed lattice plane fringes that will be discussed in more detail below. In close neighbourhood there occur examples of the smallsized background particles, see arrows in Fig. 1(b).

The rod-like particles are deposited with their long axes parallel to the substrate plane. Rotation around this axis by means of the microscope tilting-stage reveals two characteristic image contrast patterns which both have a rotational periodicity of $36^{\circ}$, i.e. backward and forward rotations lead to the same result. One of them, corresponding to orientation $A$, exhibits a symmetric contrast pattern with respect to the long axis, as shown in Fig. 2(a), and the other one, corresponding to orientation $B$, is separated from $A$ by $18^{\circ}$ rotation and exhibits a non-symmetric contrast pattern, as shown in Fig. 2(b). The characteristic image contrast patterns result from different lattice planes contributing to the image which is reflected by the corresponding diffractogram. From HREM images of both orientations information on the spatial extension of the involved lattice planes may be extracted. At first sight, in orientation $A\left\{\begin{array}{llll}1 & 1 & 1\end{array}\right\}$ lattice plane fringes and certain Moiré fringes may clearly be recognised and their spacings $d_{111}$ and $d_{\mathrm{M}}$ can be measured. $\left\{\begin{array}{llll}2 & 0 & 0\end{array}\right\}$ lattice plane fringes, clearly
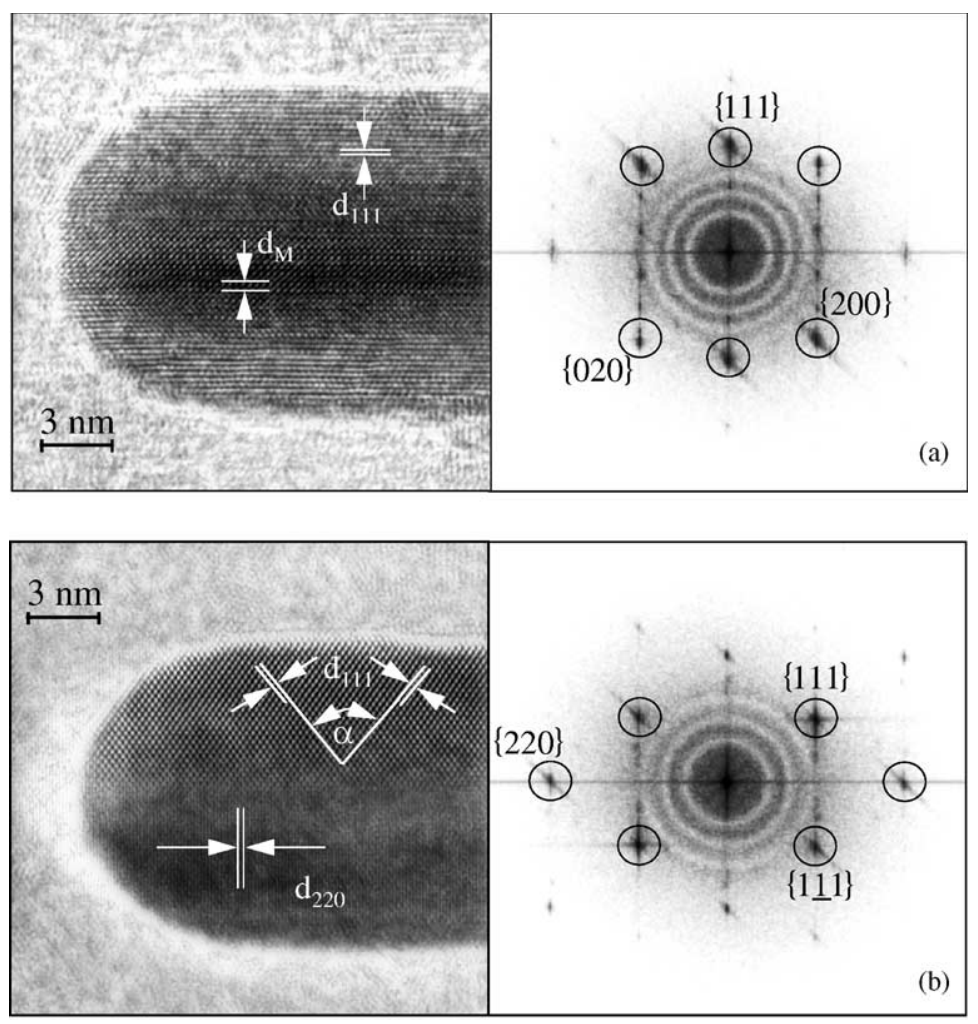

Fig. 2. Characteristic image contrast patterns obtained upon rotation around the long axis of a rod-like particle supplemented by corresponding diffractograms, where (a) shows the symmetric contrast pattern corresponding to orientation $A$, and (b) shows the nonsymmetric one corresponding to orientation $B$. 
visible by their reflections in the diffractogram, are somewhat hidden in the image. This superposition pattern will be discussed below in more detail. For orientation $B$ the situation is rather clear: the upper half exhibits two sets of $\left\{\begin{array}{lll}1 & 1\end{array}\right\}$ fringes as being characteristic of an fcc lattice in $\langle 110\rangle$ zone axis orientation, and the lower half exhibits one set of $\{220\}$ fringes perpendicular to the long axis. This contrast pattern is mirrored upon $36^{\circ}$ rotation around the rod axis.

\section{Discussion}

The peculiar contrast pattern of superimposed lattice plane fringes of the rod-like particle in orientation $A$, the 'split into two half's' contrast of such particles being in orientation $B$, and finally the rotational periodicity of the contrast patterns [23], unambiguously support the assumption that these silver nanorods are not single crystalline, but composed of subunits that are arranged around a common fivefold axis and situated in twin relation to each other. Consequently, we will discuss our experimental findings in the frame of a pentagonal rod-like MTP model as shown in Fig. 3. A pentagonal rod-like MTP is a $\left(\begin{array}{lll}0 & 0 & 1\end{array}\right)$ truncated decahedron, that may be even better described by the term 'pyramid capped pentagonal prism', which points to the anisotropic growth along the prism axis. When it is situated with the rod axis parallel to a substrate, we may distinguish 'base' (that is $A$ ) and 'side' (that is $B$ ) orientation. The 'base' orientation is that one where the electron beam runs perpendicular to one of the prism faces (one is shaded in Fig. 3) and in the 'side' orientation the electron beam runs parallel to one of these faces.

In orientation $B$, there is the lattice of the 'side' subunit, having its prism face parallel to the electron beam, situated in a $\langle 110\rangle$ zone axis orientation such that two sets of $\left\{\begin{array}{lll}1 & 1\end{array}\right\}$ fringes may be imaged in one half of the rod. Two adjacent subunits are in nearly $\langle 511\rangle$ zone axis orientation without lattice fringe imaging, and two opposed subunits are in $\langle 111\rangle$ zone axis orientation giving rise to three sets of $\{220\}$ fringes in the other half of the rod, one of them coinciding with that of the 'side' subunit. Imaging of the latter is the least sensitive to slight misorientations of the nanorod, thus mostly only this one can be seen there.

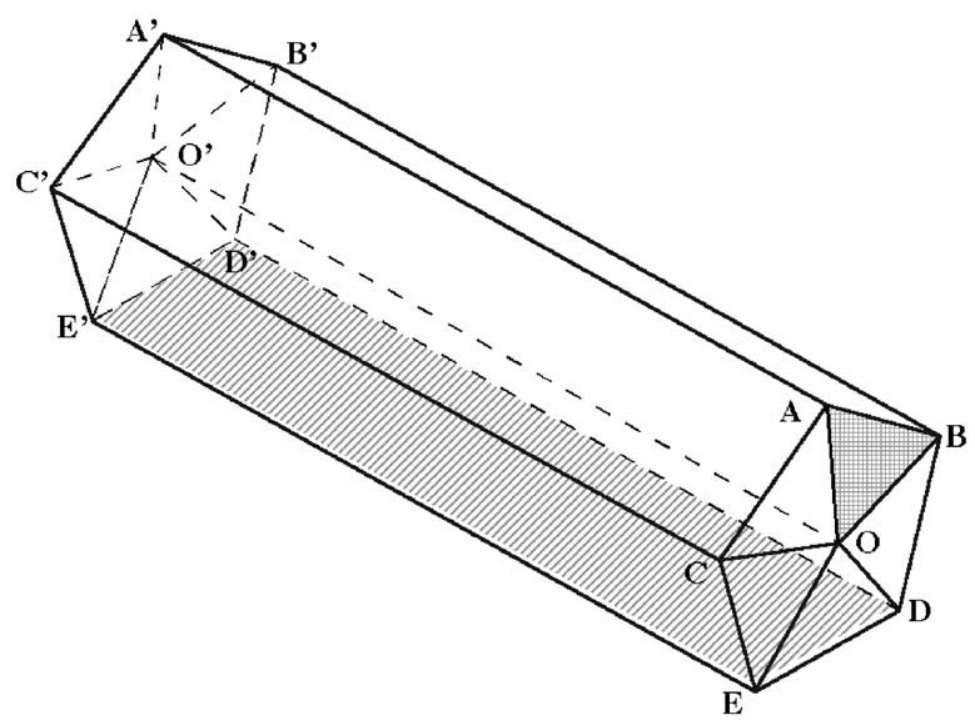

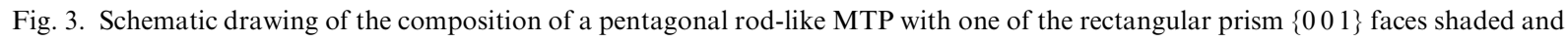
one of the triangular $\left\{\begin{array}{llll}1 & 1 & 1\end{array}\right\}$ capping faces cross-hatched. 
To understand the more complicated situation of orientation $A$ it is helpful to have a detailed look at the corresponding diffractogram shown in Fig. 4. It consists of the superposition of contributions from different subunits. The 'base' subunit is situated in a $\langle 001\rangle$ zone axis orientation giving rise to reflections marked by circles in a dashdotted line net. The spatial extension of this subunit is limited to an axial region of the rod and only there the corresponding $\{200\}$ fringes will be visible. The two adjacent subunits are situated in nearly $\langle 233\rangle$ zone axis orientation without lattice fringe imaging, and the two opposed subunits above are in $\langle 112\rangle$ zone axis orientation giving rise to reflections marked by

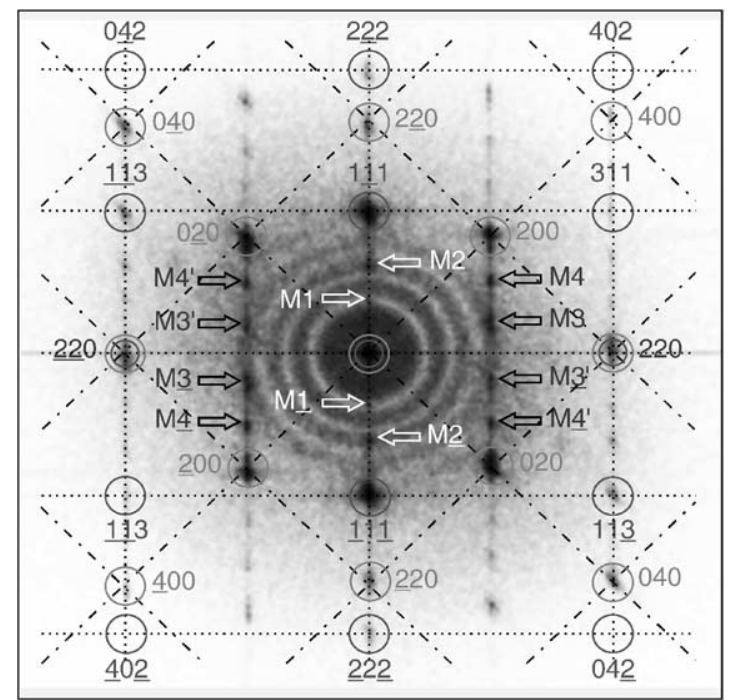

Fig. 4. Diffractogram of the rod-like particle of 1(b) with two sets of reflections marked by circles placed on a dotted line net and a dash-dotted line net, respectively, and extra reflections arranged on lines perpendicular to the rod axis (see arrows). circles in a dotted line net. The corresponding $\left\{\begin{array}{lll}1 & 1 & 1\end{array}\right\}$ fringes will be visible across the whole particle. The extra reflections arranged on lines perpendicular to the reciprocal rod axis direction, that is the vertical direction in the diffractogram shown, are due to various Moiré fringes resulting from double-diffraction effects because of superposition of subunits. We find reflections of four types of Moiré fringes M1, M2, M3 and M4, but only M1 fringes may clearly be recognised in the images of superimposed subunits such as Fig. 1(b). Parallel Moiré fringes as M1 and M2 are considered to result from double-diffraction at subunits having parallel lattice planes of different spacings, and mixed Moiré fringes as M3, M3*, $\mathrm{M}^{\#}{ }^{\#}$ and M4, M4 ${ }^{\#}$ result from double-diffraction at subunits having a certain azimuthal rotation between lattice planes of different spacings. The fringe spacing may be calculated according to commonly used equations [28]. Table 1 comprises spacings and lattice planes involved of Moiré fringes that are expected to occur by doublediffraction at $\operatorname{Ag}(001)$ and $\operatorname{Ag}\left(\begin{array}{lll}1 & 12\end{array}\right)$ fcc lattice subunits aligned along common $\{220\}$ planes. It also contains calculations for alternative combinations of lattice vectors and even Moiré vectors.

The spatial arrangement of the nanorod subunits in orientation $A$ may be elucidated by Fourier-filtered image reconstruction. This method is very helpful in separating superimposed contributions to the image [29]. By using only reflections of a $\langle 001\rangle$ zone axis orientation, marked by dash-dotted lines in Fig. 4, the reconstruction, shown in Fig. 5(a) reveals that the lattice of this subunit is restricted to only an axial stripe of the particle. As can be seen from Fig. 5(b), reconstruction by using only reflections of a (1 112$)$ lattice orientation, marked by dotted lines in

Table 1

Spacings and lattice planes involved of Moiré fringes by double diffraction at superimposed $\operatorname{Ag}\left(\begin{array}{lll}0 & 0 & 1\end{array}\right)$ and $\operatorname{Ag}\left(\begin{array}{lll}1 & 1\end{array}\right)$ fcc lattice subunits

\begin{tabular}{llllllll}
\hline Moiré type & M1 parallel & M2 parallel & M3 mixed & M3* mixed & M3 ${ }^{\#}$ mixed & M4 mixed & M4 mixed \\
\hline Planes involved & 222 and 220 & 111 and 220 & 111 and 200 & M2 and 200 & M1 and M3 & M1 and 200 & M2 and M3 \\
Spacing (nm) & 0.6420 & 0.3729 & 0.2820 & 0.2820 & 0.2794 & 0.2532 & 0.2500 \\
\hline
\end{tabular}



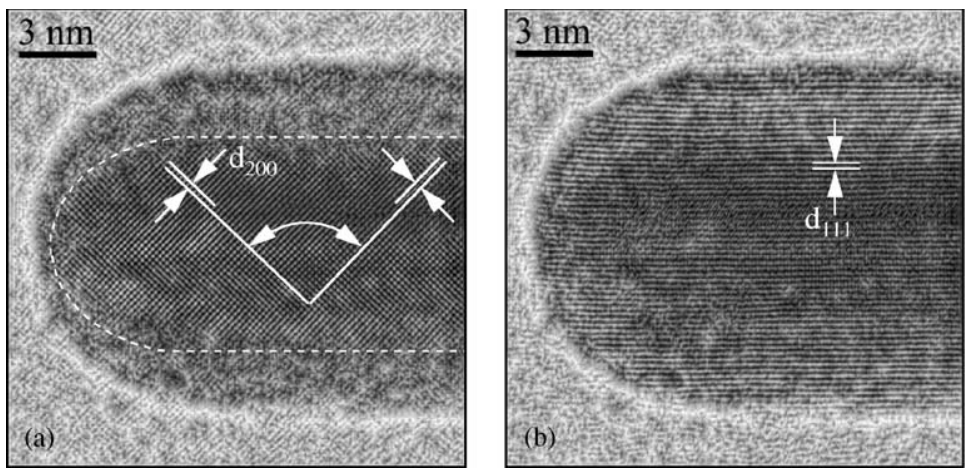

Fig. 5. Fourier filtered image reconstruction of the particle of 1(b) with (a) using only the reflections of a (00 1) lattice orientation, and (b) only those of a ( $\left.\begin{array}{lll}1 & 1 & 2\end{array}\right)$ lattice orientation, respectively.

Fig. 2, gives a uniform appearance of $\left\{\begin{array}{lll}1 & 1 & 1\end{array}\right\}$ lattice plane fringes across the whole particle.

Imaging of lattice plane fringes as shown in the above figures allows to determine the fringe spacings and angular relations of various types of lattice planes and Moiré fringes in nanorods of both orientations. Such measurements may help to find out if the space-filling property of the lattice of the multiply twinned nanorods under study is brought about by inhomogeneous lattice distortions [30] or by a transition to a homogeneously distorted fcc lattice that corresponds to a basecentred orthorhombic (bco) point group symmetry [31]. The issue of space-filling is due to the angle deficit of about $7.5^{\circ}$ that must nominally occur when stacking twin-related fcc subunits around a common axis. A transition to the bco lattice is achieved by applying biaxial stress so as to stretch the direction parallel to the fivefold axis and to compress the radial direction [31]. This slight deviation from the fcc lattice results in $72^{\circ}$ twin angle and therefore no deficit occurs. Experimentally, however, there is strong evidence of inhomogeneous strains and also of stress relief, either by lattice defects $[32,33]$ or by tetragonal lattice distortion of one to three MTP subunits [34,35].

The spacings of lattice planes derived from the fcc lattice may differ in the bco lattice with respect to their orientation to the fivefold axis. Consequently, the $\left\{\begin{array}{lll}1 & 1 & 1\end{array}\right\}$ planes (fcc notation), e.g., imaged at nanorods in $A$ orientation should have smaller spacing than those of nanorods in $B$ orientation. Thus, we measured the spacings of $\{111\},\{200\}$ and $\{220\}$ lattice plane fringes of a number (up to 20) of nanorod subunits in the various zone axis orientations. The results of this evaluation are presented in Table 2 together with the respective fcc bulk values and those of a bco lattice model. For comparison, we added also the difference between average of the measured values and fcc bulk value, as well as bco value. This difference is smaller only in two cases, but mostly larger, when assuming a bco lattice model instead of the fcc lattice. If we replace in this table the bulk value of M4 by that of M4 ${ }^{\#}$ (see Table 1) we may state that all measured fringe spacings within the experimental uncertainty agree with the corresponding bulk values. The results of this table unambiguously confirm that the lattice of the pentagonal nanorod subunits is of fcc type.

The evaluation of angular relations between lattice plane fringes, that may also differ depending on what type of lattice is considered, lead to similar results. For the angle $\alpha$ between two sets of $\left\{\begin{array}{lll}1 & 1\end{array}\right\}$ planes of nanorods in $B$ orientation, see Fig. 2(b), we measured values ranging from $70.19^{\circ}$ to $72.75^{\circ}$ yielding a mean of $70.89^{\circ} \pm 0.72^{\circ}$. The corresponding fcc bulk value is $70.53^{\circ}$ and the value of the bco lattice model amounts to $74.76^{\circ}$. Accordingly, we found for the angle $\beta$ between two sets of $\{200\}$ planes of nanorods in $A$ orientation, see Fig. 5(a), values ranging from $89.41^{\circ}$ to $91.83^{\circ}$ yielding a mean of $90.60^{\circ} \pm 0.72^{\circ}$ while the corresponding fcc bulk value is $90^{\circ}$ and the bco 
Table 2

Comparison of measured (pentagonal nanorods), tabulated (fcc) and calculated (bco) lattice fringe spacings of silver

\begin{tabular}{|c|c|c|c|c|c|c|}
\hline $\begin{array}{l}\text { Fringe type and } \\
\text { zone axis }\end{array}$ & $\begin{array}{l}\text { Spacing } \\
(\mathrm{nm})\end{array}$ & $\begin{array}{l}\text { Std. dev. } \\
(\mathrm{nm})\end{array}$ & $\begin{array}{l}\text { Bulk value } \\
(\mathrm{nm})\end{array}$ & $\begin{array}{l}\text { Difference } \\
(\%)\end{array}$ & $\begin{array}{l}\text { bco value } \\
(\mathrm{nm})\end{array}$ & $\begin{array}{l}\text { Difference } \\
(\%)\end{array}$ \\
\hline$\left\{\begin{array}{lll}1 & 1 & 1\end{array}\right\}\left\langle\begin{array}{lll}1 & 1 & 2\end{array}\right\rangle$ & 0.2348 & 0.0044470 & 0.2359 & -0.470 & 0.2339 & 0.380 \\
\hline$\{220\}\langle 112\rangle$ & 0.1446 & 0.0005624 & 0.1445 & 0.069 & 0.1520 & -4.870 \\
\hline$\left\{\begin{array}{llll}2 & 0 & 0\end{array}\right\}\left\langle\begin{array}{llll}0 & 0 & 1\end{array}\right\rangle$ & 0.2050 & 0.0014710 & 0.2044 & 0.290 & 0.2095 & -2.150 \\
\hline$\left\{\begin{array}{lll}2 & 2 & 0\end{array}\right\}\left\langle\begin{array}{llll}0 & 0 & 1\end{array}\right\rangle$ & 0.1452 & 0.0011210 & 0.1445 & 0.480 & 0.1445 & 0.480 \\
\hline$\left\{\begin{array}{lll}1 & 1 & 1\end{array}\right\}\left\langle\begin{array}{lll}1 & 1 & 0\end{array}\right\rangle$ & 0.2341 & 0.0021400 & 0.2359 & -0.770 & 0.2416 & -3.10 \\
\hline$\left\{\begin{array}{lll}2 & 0 & 0\end{array}\right\}\langle 110\rangle$ & 0.2041 & 0.0023300 & 0.2044 & -0.150 & 0.1990 & 2.560 \\
\hline$\{220\}\langle 110\rangle$ & 0.1444 & 0.0004912 & 0.1445 & 0.069 & 0.1445 & -0.069 \\
\hline$\{220\}\langle 111\rangle$ & 0.1438 & 0.0003214 & 0.1445 & 0.480 & 0.1443 & -0.350 \\
\hline$\{\mathrm{M} 1\}$ & 0.6366 & 0.0191100 & 0.6420 & -0.840 & 0.6134 & 3.780 \\
\hline$\{\mathrm{M} 2\}$ & 0.3826 & 0.0084630 & 0.3792 & 0.900 & 0.3781 & 1.190 \\
\hline$\{\mathrm{M} 3\}$ & 0.2800 & 0.0017020 & 0.2820 & -0.710 & 0.2896 & -3.310 \\
\hline$\{\mathrm{M} 4\}$ & 0.2458 & 0.0046880 & 0.2532 & -2.920 & 0.2632 & -6.610 \\
\hline
\end{tabular}

lattice model value $92.88^{\circ}$. Consequently, we may notice that also the observed angular relations between lattice planes of the nanorods on average point to the fcc lattice structure being present.

From visual inspection at high magnification of HREM images of nanorods in $B$ orientation, that is where the subunit in $\langle 110\rangle$ zone axis orientation bounded by a $\left\{\begin{array}{lll}1 & 0 & 0\end{array}\right\}$ prism face is imaged in profile, frequently the impression of a certain extent of surface buckling and non-regular lattice distortions occur. This apparently results in a widening of atomic layers near the very surface and a certain waviness of $\{200\}$ planes parallel to the rod axis. To illustrate this observation we used an image processing routine ${ }^{2}$ to mark by + signs the centres of atomic columns imaged end-on as bright dots in the selection of a corresponding image. This is shown in Fig. 6 where the $\{100\}$ surface runs nearly horizontally in the middle of the upper part. Some arrows point to regions of obvious lattice distortion. In the lower part a graphic representation of this situation is given where the atomic columns are marked by circles. A net of lines connecting the centres of these circles may demonstrate lattice distortions and lattice plane waviness more distinctly.

\footnotetext{
${ }^{2}$ Peak Analysis of the NCEM Image Processing Custom Function Plug-Ins for Digital Micrograph (http://ncem.lbl.gov/ frames/software.htm)
}

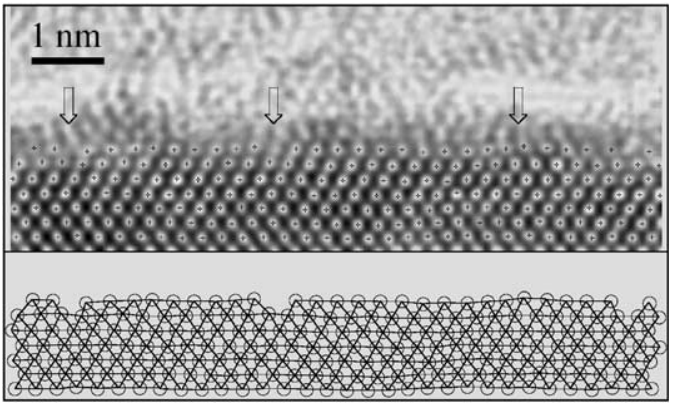

Fig. 6. Surface profile image of a pentagonal nanorod in $B$ orientation with the centres of atomic columns marked by +signs (top), and schematic representation of this area with atomic columns marked by circles whose centres are connected by straight lines (bottom). The arrows indicate regions of obvious lattice distortion.

Evidence of lattice distortions has been reported repeatedly for single-crystalline and twinned nanorods of gold that include rearrangement of surface atoms, surface relaxation, surface steps and surface reconstruction [36,37]. Such distortions are apparently required for stability reasons. At this point of the investigation we may not argue about a quantification of the observed lattice distortions. This will require more detailed evaluation and even contrast calculation. However, as long as fcc spacings and angular relations measured on average and no extended defects are recognised, there should be present a certain 
extent of lattice distortion to achieve space-filling property.

\section{Conclusions}

Multiply twinned nanorods attract much interest because of several reasons. One is the shape anisotropy enabling severe changes in surfacesensitive properties. Another is the issue of their composition and lattice structure which should be sensitive somehow to shape changes. We found that silver nanorods prepared by inert-gas aggregation have the characteristic shape of a pentagonal prism capped by pentagonal pyramids on both ends. A lattice analysis by digital image processing and evaluation revealed no deviation from fcc structure. Instead of extended lattice defects that may help to master the lack in space filling due to the particle composition we observed non-regular lattice distortions beneath the prism surfaces. As an open question there remains the issue of factors controlling the anisotropic growth of these nanorods.

\section{Acknowledgements}

The authors would like to thank J. Urban for critical discussion and $\mathrm{K}$. Weiss for technical assistance.

\section{References}

[1] L.D. Marks, Phil. Mag. A 49 (1984) 81.

[2] A. Howie, L.D. Marks, Phil. Mag. A 49 (1984) 95.

[3] P.M. Ayajan, L.D. Marks, Phase Trans. 24-26 (1990) 229.

[4] H. Hofmeister, Cryst. Res. Technol. 33 (1998) 3.

[5] S.A. Nepijko, H. Hofmeister, H. Sack-Kongehl, R. Schlögl, J. Crystal Growth 213 (2000) 129.

[6] B. Pauwels, D. Bernaerts, S. Amelinckx, G. van Tendeloo, J. Joutsensaari, E.I. Kauppinen, J. Crystal Growth 200 (1999) 126.

[7] L.D. Marks, Rep. Prog. Phys. 57 (1994) 603.
[8] A.J. Melmed, D.O. Hayward, J. Chem. Phys. 31 (1959) 545.

[9] F. Ogburn, B. Paretzkin, H.S. Peiser, Acta Cryst. 17 (1964) 774.

[10] R.W. DeBlois, J. Appl. Phys. 36 (1965) 1647.

[11] R.W. DeBlois, J. Vac. Sci. Technol. 3 (1966) 146.

[12] C. Digard, M. Maurin, J. Robert, Met. Corros. Ind. 51 (1976) 255, 320 .

[13] T. Hayashi, T. Ohno, S. Yatsuya, R. Uyeda, Jpn. J. Appl. Phys. 16 (1977) 705.

[14] M. Brieu, M. Gillet, Thin Solid Films 100 (1983) 53.

[15] M. Brieu, M. Gillet, Thin Solid Films 167 (1988) 149.

[16] M. Gillet, M. Brieu, Z. Phys. D 12 (1989) 107.

[17] B.C. Smith, P.L. Gai, Proceedings of the Eighth European Congress Electron Microsc., Programme Comm. 8. European Congress EM, Budapest, 1984, Vol. 2, p. 1151.

[18] U. Dahmen, K.H. Westmacott, Science 233 (1986) 875.

[19] T.N. Millers, A.A. Kuzjukévics, Prog. Cryst. Growth Charact. 16 (1988) 367.

[20] M. Arita, N. Suzuki, I. Nishida, J. Crystal Growth 132 (1993) 71.

[21] A.E. Romanov, I.A. Polonsky, V.G. Gryaznov, S.A. Nepijko, T. Junghanns, N.I. Vitrykhovski, J. Crystal Growth 129 (1993) 691.

[22] I. Lisiecki, A. Filankembo, H. Sack-Kongehl, K. Weiss, M.-P. Pileni, J. Urban, Phys. Rev. B 61 (2000) 4968.

[23] S.A. Nepijko, D.N. Ievlev, W. Schulze, J. Urban, G. Ertl, Chem. Phys. Chem. 3 (2000) 140.

[24] J.P. Wilcoxon, J.E. Martin, F. Parsapour, B. Wiedemann, D.F. Kelly, J. Chem. Phys. 108 (1998) 9137.

[25] M.B. Mohamed, V. Volkov, S. Link, M.A. El-Sayed, Chem. Phys. Lett. 317 (2000) 517.

[26] F. Frank, W. Schulze, B. Tesche, J. Urban, B. Winter, Surf. Sci. 156 (1985) 90.

[27] W. Rasband, NIH Image, public domain software, US National Institute of Health (http://rsb.info.nih.gov/nihimage/)

[28] J.W. Edington, Practical Electron Microscopy in Materials Science, TechBooks, Herndon, 1976.

[29] J.C. Russ, The Image Processing Handbook, CRC Press, Boca Raton, 1995.

[30] J. Yang, J. Crystal Growth 47 (1979) 274.

[31] A. Howie, L.D. Marks, Phil. Mag. A 49 (1984) 95.

[32] L.D. Marks, D.J. Smith, J. Microsc. 130 (1983) 249.

[33] H. Hofmeister, T. Junghanns, Mater. Sci. Forum 113-115 (1993) 631.

[34] Q. Chen, M. Tanaka, K. Furuya, Surf. Sci. 440 (2000) 398.

[35] Y. Wu, Q. Chen, M. Takeguchi, K. Furuya, Surf. Sci. 462 (2000) 203.

[36] Z.L. Wang, R.P. Gao, B. Nikoobakht, M.A. El-Sayed, J. Phys. Chem. B 104 (2000) 5417.

[37] M.J. Yacamán, J.A. Ascencio, G. Canizal, Surf. Sci. 486 (2001) L449. 